- The retruded contact position (RCP) is a relatively reproducible maxillomandibular relationship.

- It is used as a reference point for mounting casts on an articulator.

- Occlusion has a biological adaptability and is not constant.

VERIFIABLE

- Mandibular guidance from the operator has been shown to give more consistent

RCP recordings.

- Chin point guidance, bimanual manipulation and use of an anterior jig are recommended.

\title{
Recording the retruded contact position: a review of clinical techniques
}

\author{
P. H. R. Wilson ${ }^{1}$ and A. Banerjee ${ }^{2}$
}

\begin{abstract}
The retruded contact position (RCP) is an important maxillomandibular relation in restorative dentistry. This review will describe RCP and consider its importance and use in the restorative dental treatment of the dentate and edentulous subject. A comparative account of the various mandibular guidance methods used to obtain a RCP record will be presented, followed by recommendations for fixed and removable clinical prosthodontic practice.
\end{abstract}

The three-dimensional relationship of the mandible to the maxilla, and the clinician's understanding of it, is fundamental in clinical dental practice. No matter the degree of restorative dental treatment provided, be it a small occlusal restoration to a full-mouth rehabilitation, the occlusion is affected to a greater or lesser extent. Occlusion has been defined simply by Davis and Gray ${ }^{1}$ as 'the contacts between teeth'. These contacts can be considered statically or dynamically, as teeth slide over each other during mandibular movement. In addition to the occlusion, the masticatory system is also composed of the periodontal ligaments, the temporomandibular joints (TMJ), the muscles of mastication and their associated ligaments. ${ }^{2}$ The system is under the control of higher centres in the central nervous system.

The interpretation of various mandibular positions is fraught with confusion ${ }^{3-6}$ as over the past 150 years there have been a succession of occlusal schools of

1"Specialist Registrar in Restorative Dentistry ${ }^{2}$ Lecturer/Honorary Specialist Registrar in Restorative Dentistry, Guy's \& St Thomas' Hospital NHS Trust, Unit of Restorative Dentistry, Floor 26, Guy's Hospital, London Bridge, London SE1 9RT

${ }^{*}$ Correspondence to: Paul Wilson

Email: paul.wilson@gstt.sthames.nhs.uk

\section{Refereed Paper}

doi:10.1038/sj.bdj.4811130

Received 09.01.03; Accepted 14.05.03

๑ B British Dental Journal 2004; 196: 395-402 thought. ${ }^{7}$ The early Conical ${ }^{8}$ and Spheri$\mathrm{cal}^{9}$ theories were superseded by the mechanical models of the Gnathologists. ${ }^{10}$ These theories were largely driven by developments in articulator design. As a consequence, the terminology, teaching, clinical methods and research in this important area has left many bewildered. The Glossary of Prosthodontic Terms has contributed to this confusion by periodically altering the definitions of certain relations. ${ }^{11,12}$ In recent years, the engineering model of occlusion has been tempered by an increased appreciation of the biological aspects of the masticatory system. ${ }^{7}$ Biological occlusion does not discard the important work undertaken over the past 150 years, but recognises the interaction between the component parts of the masticatory system and the fact there is a degree of adaptability. ${ }^{7}$

This review will describe RCP and consider its importance and use in restorative dental treatment of the dentate and edentulous subject. A comparative account of the various mandibular guidance methods used to obtain an RCP record will be presented, followed with recommendations for fixed and removable clinical prosthodontic practice.

\section{THE RETRUDED CONTACT POSITION OF THE MANDIBLE \\ Terminology}

The retruded contact position (RCP; Table1 and Fig. 1) is also variously known as the premature contact in centric relation, ${ }^{2}$ centric relation contact position (CRCP), the retruded axis position (RAP), the terminal hinge position (THP) or the ligamentous position. Terminology, as alluded to earlier, is very confusing as The Glossary of Prosthodontic Terms ${ }^{11,12}$ has changed definitions from edition to edition, and has even cited definitions which appear contradictory. Moreover, there are seven different definitions for centric relation (CR) in use (Table 2). Jasinevicius et al. ${ }^{14}$ surveyed the level of consensus regarding the definition of CR in seven US dental schools. The authors found that numerous definitions were used at each institution and the longer a clinician had been qualified for correlated with which definition they employed. It was suggested that this had an adverse effect on undergraduate dental student's understanding of CR, even diminishing the value which they placed on CR. While the debate over the semantics of $\mathrm{CR}$ continues amongst academic dentists, many clinicians still consider centric occlusion (CO) as a synonym for the intercuspal postion (ICP, Table 1). This is obviously incorrect if the Glossary of Prosthodontic Terms is to followed, but until terminology and teaching issues are resolved and consensus is reached, this concept will probably persist. We do not wish to contribute to the confusion, and to this end we will follow the definitions reproduced in Table 1 and the most recent definition of CR in Table 2 (Definition No. 1). 


\section{PRACTICE}

\begin{tabular}{|c|c|c|}
\hline Term & Abbreviation & Definition \\
\hline $\begin{array}{l}\text { Retruded contact } \\
\text { position }\end{array}$ & $\mathrm{RCP}$ & $\begin{array}{l}\text { Guided occlusal relationship occurring at the } \\
\text { most retruded postion of the condyles in } \\
\text { the joint cavities. A position that may be } \\
\text { more retruded than the centric } \\
\text { relation position. }\end{array}$ \\
\hline Intercuspal position & ICP & $\begin{array}{l}\text { The complete intercuspation of the opposing } \\
\text { teeth independent of condylar position. } \\
\text { Sometimes referred to as the best fit of } \\
\text { the teeth regardless of the condylar } \\
\text { position. }\end{array}$ \\
\hline Centric occlusion & $\mathrm{CO}$ & $\begin{array}{l}\text { The occlusion of opposing teeth when the } \\
\text { mandible is in centric relation. This may } \\
\text { or may not coincide with the intercuspal } \\
\text { position. }\end{array}$ \\
\hline
\end{tabular}

\section{The importance of RCP}

RCP is said to be a relatively reproducible position $^{15-18}$ and as such is useful in the restorative management of dentate and edentulous individuals and as a reference point for the registration of transfer records, so that casts can be mounted on articulators. Posselt ${ }^{15}$ in his classic treatise 'Studies in the Mobility of the Human Mandible', found that the retruded position of the mandible was reproducible to within $0.08 \mathrm{~mm}$ and thus could be termed a border movement. This reproducibility is achieved by virtue of the non-elastic nature of the temporomandibular joint capsule and the associated capsular ligaments. The influence of mandibular guidance on RCP reproducibility will be discussed later.

\section{Dentate subjects}

In the dentate patient, the RCP is an unstrained position of the mandible relative to the maxilla occurring at initial tooth contact(s). This contact follows closure about the terminal hinge axis (Fig. 1) where the condylar heads are in their most anterior and superior position in the glenoid fossae (Fig. 2). In this position the condylar heads are fully seated in the glenoid fossae, with the thinnest, avascular part of the fibro-elastic temporomandibular joint disc interposed. Posselt in $1952^{15}$ found that in 10\% of dentate individuals, the RCP coincided with the intercuspal position (ICP; Table 1; common synonyms include: maximum intercuspation (MI) or centric occlusion (CO)). For the remainder, the RCP is infero-posterior to ICP by 0.5-2 $\mathrm{mm}$. The movement from the RCP to the ICP is known as a slide ${ }^{19}$ (Fig. 1). A slide has the potential for a combination of horizontal, vertical and lateral components along its path.

The situation for partial dentures falls between that for dentate patients and edentulous patients. Important factors include the distribution of natural teeth, distribution of natural occlusal contacts and the patient's existing occlusal vertical dimension. ${ }^{19}$ Subjects with an easily identifiable, stable and comfortable ICP may only require a conformative approach rather than reorganisation at RCP. Reorganisation involves altering a patient's existing ICP to a new ICP. This new ICP is made coincident with RCP because of the reproducibility of the latter. This will eliminate the RCP-ICP slide.

\section{Edentulous subjects}

In the edentulous patient there are no natural tooth contacts to define a retruded contact. In this situation, prosthetic tooth contact (or wax occlusal rim contact) will be along the retruded arc of closure (Fig. 1) at some point. This is dictated by the occlusal vertical dimension (OVD) appropriate for that patient. Therefore, the mandible and maxilla are in CR (Table 2) at this occlusal vertical dimension and it is from here that the prosthetic occlusal scheme is constructed. Moreover, this position is also the starting point for eccentric mandibular movement. A retruded mandibular relationship is recorded due to its relative reproducibility ${ }^{15-18}$ and to produce stability of the denture bases, in conjunction with a lack of interfering contacts in eccentric mandibular positions. It may also contribute to TMJ health although no definitive evidence exists. ${ }^{20}$ Fenlon et al. ${ }^{21}$ has demonstrated a positive correlation between complete denture usage and accuracy of the $\mathrm{CR}$ registration.

\section{USES OF RCP IN THE DENTATE PATIENT}

The importance of recording the retruded mandibular position in edentulous patients has been described. ${ }^{21}$ In dentate patients, the location and reproducible recording of the RCP is important in:

- Mounting models on an articulator. Mandibular movement can be simulated because of pure rotation about the terminal hinge axis (Fig. 1). ${ }^{22}$

- Reorganising a patient's occlusion at a new occlusal vertical dimension. ${ }^{2}$

- Occlusal analysis in cases of toothwear, tooth mobility, drifting, pain or repeatedly failing restorations. ${ }^{2}$

- Occlusal splint therapy. ${ }^{23,24}$

- 'Distalising' the mandible to create palatal space for anterior restorations. ${ }^{4}$

- Restoring a tooth which is involved in determining the RCP. ${ }^{25}$

- Determining the magnitude and direction of the RCP to ICP slide in order to assess the resultant force applied to anterior restorations. ${ }^{5}$

Fig. 1 Posselt's sagittal envelope of mandibular border movements. ${ }^{15}$

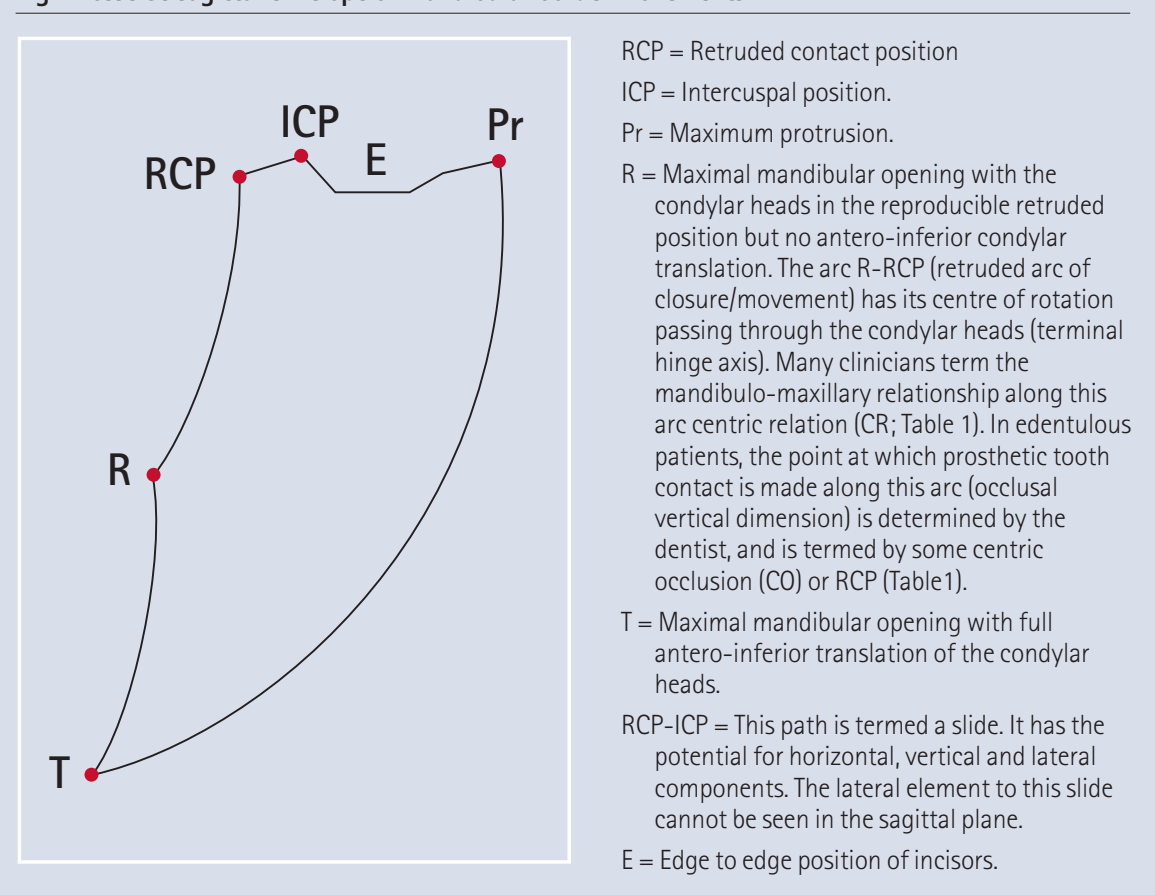


Fig. 2 Colour image showing the relevant bony anatomy of the skull base and the right TMJ articulation. A: mastoid process, B: right glenoid (articular) fossa with the antero-superior aspect shaded, C: zygomatic arch D: posterior hard palate, E: pterygoid plates, and F: styloid process

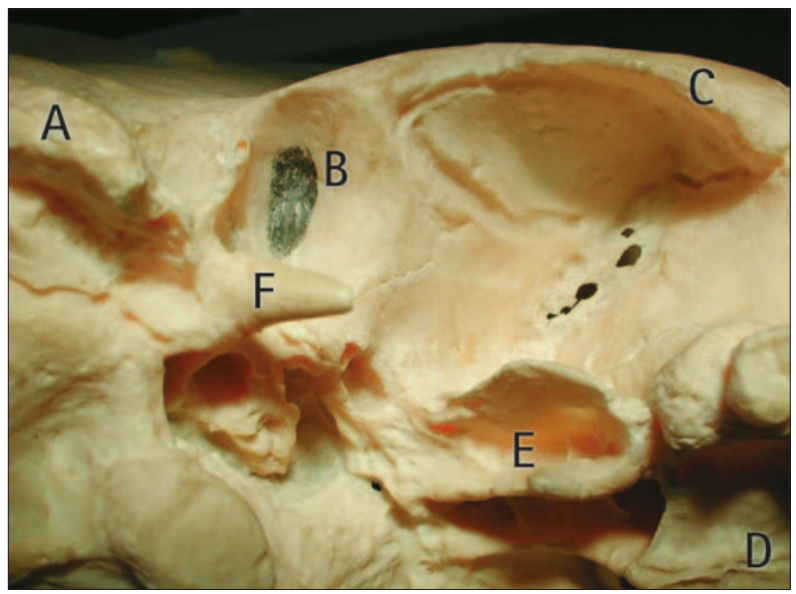

- Midline analysis in cases of facial asymmetry, in order to separate dental and

It has been suggested that RCP is attainable during mastication and deglutition, ${ }^{4}$ and that restoring a patient to this position may result in enhanced masticatory efficiency and occlusal stability. ${ }^{26}$ However, there is no published evidence for these benefits. Masticatory efficiency is a complex subject and consensus about what constitutes the ideal chewing pattern does not exist. ${ }^{27}$ Furthermore, it has been demonstrated that the reorganisation of patients to a situation where RCP coincides with ICP will relapse after a period of time so that a slide between the two is re-introduced. ${ }^{28}$ Celenza $^{28}$ re-examined 32 patients, for which he had reorganised ICP to coincide with their RCP, 2-12 years post-treatment. Thirty patients had reestablished their ICP anterior to RCP, the resulting slide being $0.02-0.36 \mathrm{~mm}$. This Unfortunately this work has not been repeated and verified.

\section{FACTORS AFFECTING THE RCP RECORDING}

Recording RCP is dependent upon a number of factors including the patient, operator experience and training (see later), the registration material and recording method employed, the time of the recording, guidance of the mandible (see later), neuromuscular conditioning and record han-

The diurnal variance of recording maxillomandibular relationships has been studied in 13 subjects by Shafagh et al. ${ }^{31}$ Many aspects of human biology are subject to circadian rhythms, including soft tissue volumes, joint space volumes, blood pressure, tooth mobility, salivary gland function, gingival crevicular fluid exudation and whole body posture. Shafagh et $a l .{ }^{31}$ found that retruded mandibular recordings made in the skeletal causes. ${ }^{26}$ was ascribed to condylar remodelling. dling and storage. ${ }^{22,29-31}$ evening were more posterosuperior than those made in the morning. Apart from tissue changes, these differences may be due to the state of patient, and therefore, masticatory muscle relaxation.

There are many registration media which can be used and they all have advantages and disadvantages. ${ }^{32}$ Media include waxes, zinc oxide pastes, acrylic resins and elastomeric materials. Warren and $\mathrm{Capp}^{33}$ recommended that the ideal recording material should demonstrate low initial viscosity, set rigidly and be dimensionally accurate and stable. It must also be unaffected by disinfection protocols, and be carefully handled and stored so as to avoid distortion. Polymeric impression materials have been shown to be the most accurate $^{34}$ but operator preference and technique are also important.

In the dentate individual the RCP record is taken at a slightly increased OVD just prior to tooth contact (CR; Fig. 1) with the mandible rotating about the terminal hinge axis. If tooth contact occurs, involuntary programmed mandibular deviation from the hinge axis will result due to sensory feedback from periodontal ligament mechanoreceptors. ${ }^{2}$ Neuromuscular conditioning and the abolition of reflex patterns of closure can be achieved by the patient biting the teeth together hard, biting on cotton rolls, holding the mouth open wide, use of an anterior jig (see later) or use of an occlusal splint. These methods will result in masticatory muscle fatigue and relaxation, thus permitting easier operator-manipulation of the mandible. ${ }^{22}$ Closing the articulated study casts through the thickness of the registration permits the identification of RCP and its relation to ICP. Errors from recording about an arbitrary hinge axis will result in the erroneous detection of tooth contacts. The average terminal hinge axis location is recorded by some facebows (eg Denar Slidematic, Denar Corp., Anaheim, USA), but the true hinge axis position
Table 2 Definitions of centric relation ${ }^{11}$

\begin{tabular}{|c|c|c|}
\hline Term & Abbreviation & Definition \\
\hline $\begin{array}{l}\text { Centric } \\
\text { relation }\end{array}$ & $C R$ & $\begin{array}{l}\text { 1. The maxillomandibular relationship in which the condyles articulate } \\
\text { with the thinnest avascular portion of their respective discs, with the } \\
\text { complex in the anterior-superior position against the slopes of the } \\
\text { articular eminences. This position is independent of tooth contact. It } \\
\text { is restricted to a purely rotary movement about the transverse } \\
\text { horizontal axis. }\end{array}$ \\
\hline
\end{tabular}

2. The most retruded physiologic relation of the mandible to the maxillae to and from which the individual can make lateral movements. It is a condition that can exist at various degrees of jaw separation. It occurs around the terminal hinge axis.

3. The most retruded relation of the mandible to the maxillae when the condyles are in the most posterior unstrained position in the glenoid fossae from which lateral movements can be made, at any given degree of jaw separation.

4. The most posterior relation of the lower to the upper jaw from which lateral movements can be made at a given vertical dimension.

5. A maxilla to mandible relationship in which the condyles and discs are thought to be in the midmost, uppermost postion. The position has been difficult to define anatomically but is determined clinically by assessing when the jaw can hinge on a fixed terminal axis (up to $25 \mathrm{~cm}$ ). It is a clinically determined relationship of the mandible to the maxilla when the condyle disc assemblies are positioned in their most superior position in the mandibular fossae and against the distal slope of the articular eminence.

6. The relation of the mandible to the maxillae when the condyles are in the uppermost and rearmost postion in the glenoid fossae. This position may not be able to be recorded in the presence of dysfunction of the masticatory system

7. A clinically determined position of the mandible placing both condyles into their anterior uppermost position. This can be determined in patients without pain or derangment in the TMJ. 
can be found accurately using a kinematic facebow. The latter method is time-consuming and it has been suggested that no practical advantage is gained. ${ }^{26}$

Other influences that affect the RCP recording may be difficult for the clinician to control. These include general health, attitude to treatment, co-operation and comprehension of the procedure, the patient's body, head and tongue position, state of relaxation, medication and state of anaesthesia. Only a few of these have been assessed objectively. Helkimo $^{22}$ found no difference in recordings between supine and upright patients. Pain from the operator's guidance technique, the temporomandibular joints or from muscle tension will result in reflex mandibular protrusion and hence erroneous recordings. Psychological tension and anxiety will also increase muscle tension. The number of teeth, their condition or the ridge form of edentulous patients will effect the stability of the recording medium and thus the quality of the recording.

\section{MANDIBULAR GUIDANCE \& RCP}

The aim of mandibular guidance is to help locate the condylar heads in the glenoid fossae at the terminal hinge axis in a consistent manner, thus producing mandibular closure about the terminal hinge axis. Mandibular guidance methods can be divided into those which are patient-guided and those which are operator-guided. Kaplan ${ }^{35}$ has warned against the use of excessive forces during mandibular guidance. Forceful mandibular retrusion by the operator can place the condylar heads too inferiorly, as they move down the posterior slope of the glenoid fossa and rotate about the temporomandibular ligaments. ${ }^{36}$ Furthermore, this can cause patient discomfort which results in resistance to the applied load. Some force applied by the patient, from the muscles of mastication, is beneficial when locating the condylar heads in an antero-superior position in the glenoid fossae. ${ }^{37}$ However, too much force is detrimental as the mandible flexes $^{38,39}$ about the horizontal plane.

\section{Patient-guided recording of RCP}

Schuyler technique ${ }^{40}$ (Fig. 3)

This quick and simple technique involves the patient placing the tip of the tongue to the back of the palate and closing into a horseshoe of softened wax with light pressure.

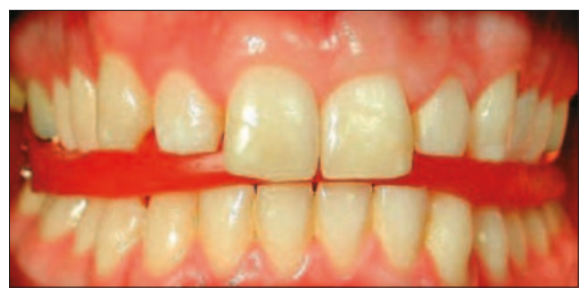

Fig. 3 Clinical view of RCP registration using the Schuyler technique. Bilayered, softened wax acts as the registration medium in this instance
Fig. 4a Maxillary and mandibular occlusal rims with a metal plate on the upper (left) and stylus on the lower (right)

Fig. 4b A close up view showing the relationship between the stylus and plate when recording $\mathrm{RCP}$ using the gothic-arch tracing method. The stylus scribes an arrow-head shaped tracing on the maxillary plate outlining the protrusive and right and left lateral excursions of the mandible. Where the lines meet on the plate represents the retruded mandibular position
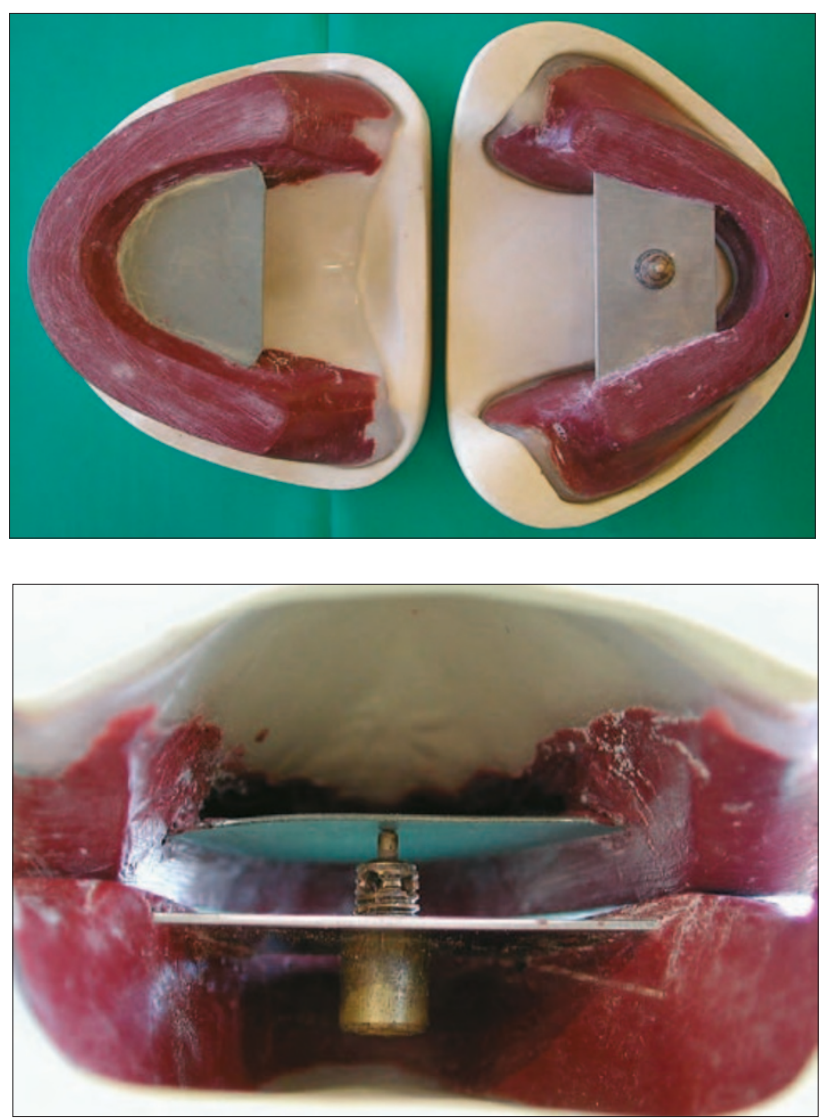

There is no way of verifying the nature of any unwanted tooth contact or the retrusion of the mandible. In addition the wax may not be uniformly softened which can lead to inaccuracies in the recording. ${ }^{34,41,42}$ This technique can also be used, with wax rims, for the edentulous patient.

\section{Physiological technique ${ }^{43}$}

This method uses cones of soft wax placed posteriorly. The patient swallows several times, simultaneously the mandible retrudes and the recording is made. Besides the uniformity of softness of the wax, there is no control over the mandibular retrusion nor any tooth contact. This technique is more appropriate for the edentulous patient.

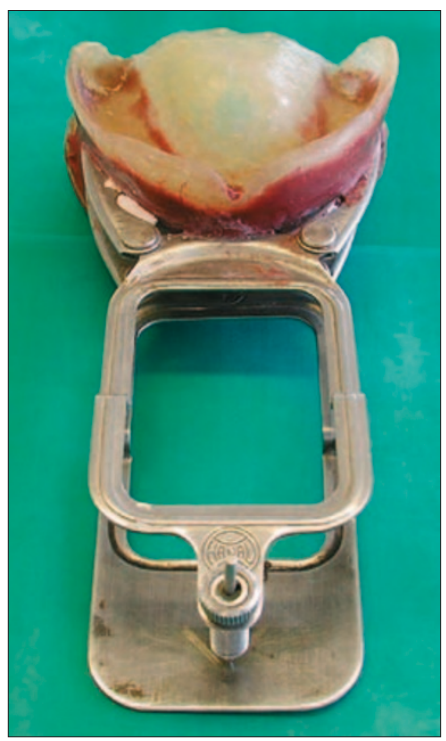

Fig. 5 A system of recording a gothicarch tracing extra-orally. The stylusplate system is attached to the rims via forks

Gothic arch (Arrow-point) tracing 44,45 (Figs 4a, 4b, 5)

This technique has been described for use in both dentate and edentulous patients. It can be used intra- or extra-orally and is based on tracing the movement of the mandible. Metal plates are added to the upper and lower wax rims. The lower plate has a central pin, which can be adjusted to the desired occlusal face height and at right angles to the opposing plate. The pin is the only point of contact between the mandible and maxilla. The patient practices mandibular excursions using the device after which a fine spray of Occlude (Pascal Co., USA) is added to the maxillary plate. The patient then replicates the excursive movements and the mandibular pin scribes an arrowhead tracing on the maxillary plate delineating the paths of these excursions. Where the three lines intersect indicates the retruded mandibular relation. 
This arrangement can be set up for an extra-oral registration following the same principles (Fig. 5). Drawbacks of this technique include the fact that it is relatively time consuming and requires well-defined, non-displaceable upper and lower alveolar ridges to allow stable and retentive acrylic bases. Large tongues can also cause base movement during the tracing. Truly reproducible excursive movements are often difficult to re-create by patients thus producing an imperfect arrowhead tracing which then requires an element of interpretation. Recently the principles of the gothic arch tracing have been revisited in the Biofunctional Prosthetic System (Ivoclar Vivadent, Germany) for complete dentures. Here the "Gnathometer 'M" consists of wax rims with tracing plates. Pantographs are mechanical or electronic devices which trace mandibular movements in a manner similar to that described above. Digital pantograph machines (ARCUS Digma, Kavo, Germany; Denar Cadiax System, Waterpik Technologies, USA) are available which are much simpler than the traditional set ups. They have an electronic facebow and lower incisal plate, which are linked remotely to a computer. Their relation to each other is measured and calculated by specialised software in real-time. RCP registrations can also be compared with each other. There are no studies using the digital systems with regard to usefulness, reliability and practicality. However, the authors propose they may be quicker and more convenient to use when compared with the traditional set-ups as they are less cumbersome and much smaller in size.

\section{Myo-monitor ${ }^{46,47}$}

The myo-monitor is an electrical jaw muscle stimulating device which is reputed to achieve muscle relaxation and produce a neuromuscular mandibular position. ${ }^{48} \mathrm{An}$ example is the J-4 Muscle Stimulator (Myotronics-Noramed Inc, USA) which produces pulsed ultra-low frequency stimulation of facial and masticatory muscles. Stimulating electrodes are placed over the coronoid notches and a common electrode is located at the nape of the neck. Proponents of the myo-monitor suggest that the 'jaw-closer' muscles act simultaneously, via reflex contraction, to produce a reproducible retruded mandibular position.

\section{Operator-guided recording of RCP} Chin-point guidance method ${ }^{49}$ (Fig. 6) The patient is seated upright and relaxed with the clinician positioned in front. A softened two-layer wax wafer $(1.4 \mathrm{~mm}$ thick) is gently pushed against the cusps of the maxillary teeth with just enough force to make slight cuspal indentations. The wafer is removed, chilled and re-seated in

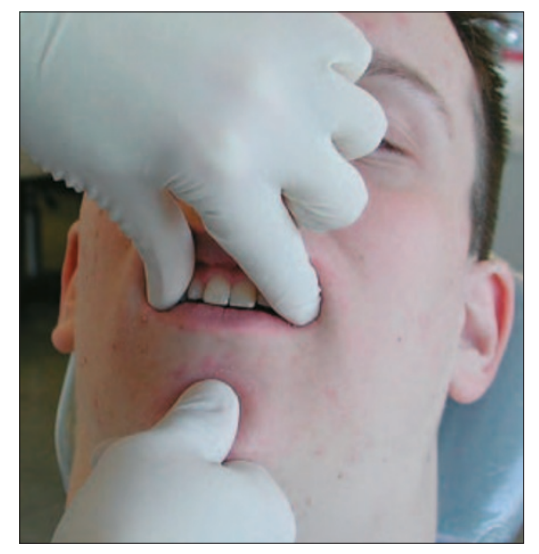

Fig. 6 A clinical extra-oral view of the chin point guidance method of recording RCP

order to check fit and stability. A registration medium is applied to the mandibular surface of the wax wafer and the patient's mandible is guided into a hinge closure by the thumb and index finger of the operator. The mandible is then manually manoeuvred a few times about the hinge axis. After several smooth movements the hinge closure is completed until the mandibular teeth just indent the registration material. The risk with this method is the ease with which the condyles can be over-retruded.

\section{Three finger chin-point guidance method 50} (Fig. 7)

This method is similar to the chin-point guidance method except for the hand position of the operator. A tripod is created at the chin-point and lower border of the mandible on both sides by the thumb, index and third finger. Gentle guidance along all three digits is required in a mid-sagittal plane. This encourages anterior-superior placement of the condyles but care is required as it is easy to deflect the mandible to one side. This technique is not recommended for edentulous subjects because the operator's hand position can lead to displacement of the lower denture base.

\section{Bimanual manipulation method ${ }^{51}$ \\ (Fig. 8)}

This technique is carried out with the patient supine and the operator seated directly behind. The fifth finger of each hand is placed behind the angle of the mandible, with the fourth fingers posi-

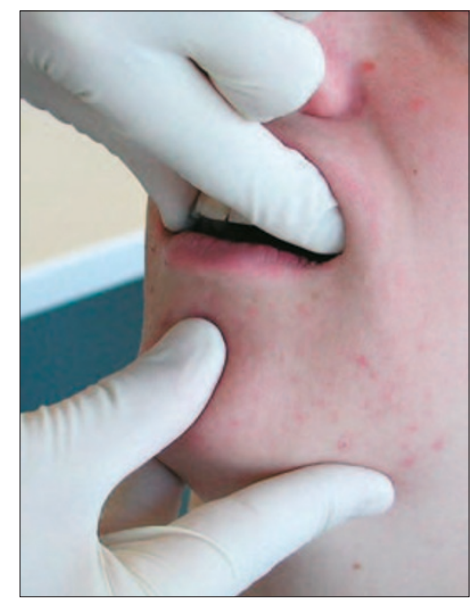

Fig. 7 A clinical extra-oral view of the three-fingered chin point guidance method. Note the difference in operator's hand position compared with Figure 6

tioned just in front of the angle. This permits the condyles to be directed anterosuperiorly within the glenoid fossae. The third fingers are placed on the inferior surface of the body of the mandible, and the index fingers submentally in the midline. The thumbs are positioned laterally to the symphysis. By opening and closing a few times on the hinge axis the patient will relax and the registration can be made. This technique can also be used for the edentulous patient assuming the lower alveolar ridge is developed enough to allow the provision of a stable and retentive lower base. An alternative method, with the operator in front of the patient, is to use the index fingers to stabilise the lower record base and guidance is from the thumbs on the chin. Smith ${ }^{52}$ has described a modification whereby one hand can stabilise both upper and lower record bases.

\section{Anterior guidance by a Lucia Jig ${ }^{53}$ (Figs} 9a, 9b)

The basis of the Lucia jig method and the techniques that follow, is to provide an anterior reference point. This forms a tripod with the condyles, helping them to locate in the most anterior-superior position in the glenoid fossae. With the teeth out of contact all proprioceptive reception from the teeth and musculature is removed. An anterior stop also stabilises the mandible during record-
Fig. 8 Bimanual manipulation method of recording RCP. The operator positions him/herself behind the supine patient and helps to gently guide the TMJ into the most antero-superior position within the glenoid fossa

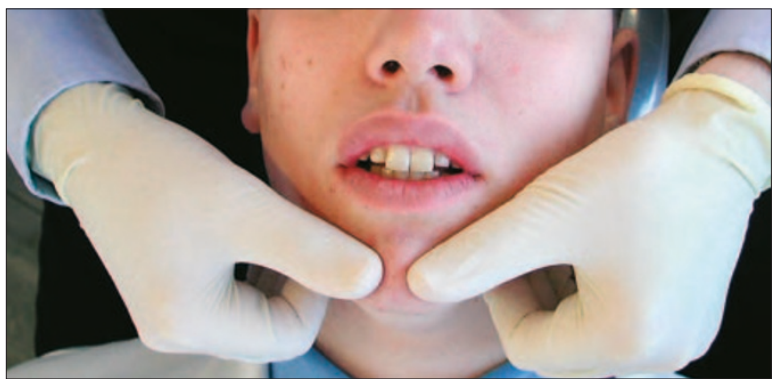




\section{PRACTICE}

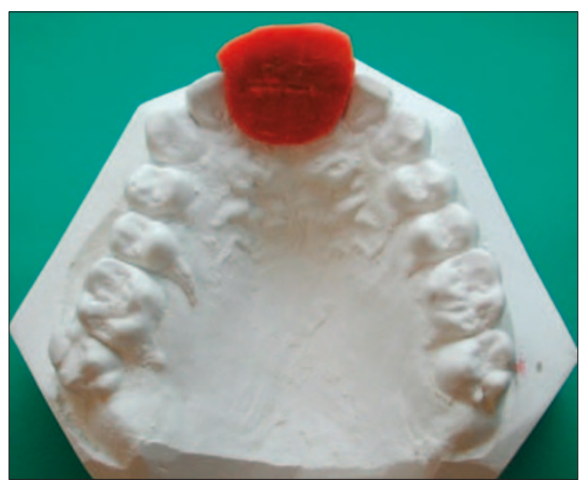

Fig. 9a A palatal view of a Lucia jig made from DuraLay on a study cast

ing and permits minimal tooth separation so that the recording medium is as thin as possible. The Lucia jig is made from self-curing acrylic resin (ie DuraLay II, Reliance Dental MFG Co., USA) on a study cast or in the mouth. If carried out intra-orally care must be taken because of the exothermic setting reaction of the acrylic. At the dough stage, the acrylic resin is adapted to the upper anterior teeth, using soft paraffin as a separator. The palatal acrylic is manipulated to just cover the palatal soft tissues. The lingual aspect should slope posteriorly and superiorly at an angle of between $40-60^{\circ}$ and a wooden spatula can be useful in achieving this. While the jig sets it must be gently taken on and off the teeth to avoid engaging undercuts and to reduce the chance of thermal trauma. Once completed the jig is adjusted using articulating paper placed on the palatal aspect whilst the patient performs lateral and antero-posterior excursive movements. A selected lower incisor scribes an arrow-head pattern, the 'wings' and 'tail' of which can be ground away to leave the apex. This process is repeated until a raised area of acrylic at the apex remains. This is the location of the retruded position and the vertical height is then adjusted until the posterior teeth are just out of contact. The record is made at this position with the jig in the mouth. It is important to note that while the jig is being adjusted out of the mouth, the patient must bite on a cotton wool roll or a saliva ejector in order to keep the teeth discluded otherwise the training effect of the jig will be lost. This method can also be used if upper anterior teeth are missing. The jig is simply made to span the edentulous area and is adjusted in the same manner.

\section{Anterior guidance by a tongue blade ${ }^{54}$ \\ (Fig. 10)}

The tongue blade method uses wooden spatulas instead of a custom made Lucia jig to provide an anterior reference point. The degree of tooth separation can be altered by the number of spatulas used. The patient's teeth must be discluded for a
Fig. 9b A clinical anterior view of the jig placed on the patient's maxillary teeth. This helps to form an anterior reference point for the registration of RCP

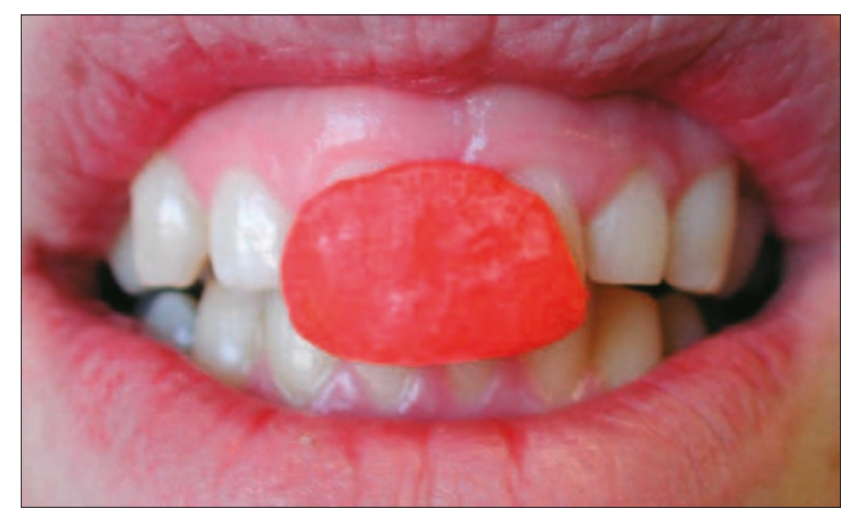

period of time, usually between 10-20 minutes prior to registration, in order for proprioceptive input to be lost. No adjustment using gothic arch principles is possible and once the correct anterior spatula guidance is achieved, registration material is used to record the relative position of the mandibular and maxillary teeth.

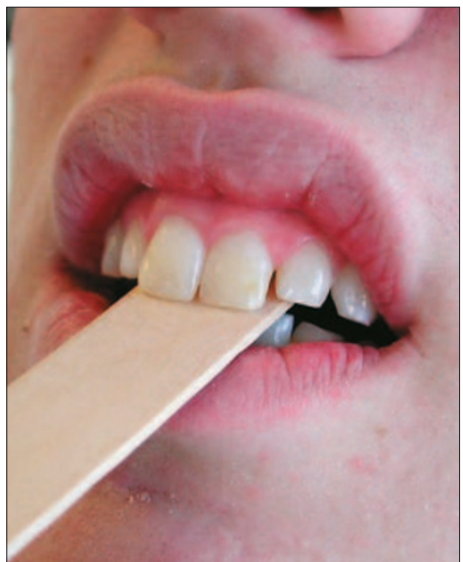

Fig. 10 An anterior clinical view showing the use of tongue blades to produce an anterior reference point during an RCP registration. Enough wooden blades are used to just achieve posterior disclusion of the teeth

Anterior guidance by a Leaf Gauge $\mathrm{e}^{55}$ (Figs 11a, 11b, 11c)

Another variation of the Lucia jig principle involves the leaf gauge. Originally, a book of ten acetate leaves was described but now disposable paper versions are also available (Panadent Corp., CA, USA). The leaves provide the anterior reference point and the degree of tooth separation can be altered until the teeth achieve disclusion. No adjustment using gothic arch principles is possible. A registration support wafer permits the registration of the inter-dental record (Fig. 11c).

\section{Anterior guidance by a OSU Woelfel} Gauge $^{56}$ (Figs 12a,12b)

This method was developed by Woelfel at Ohio State University (OSU) and aims to simplify the Lucia jig technique while still achieving an anterior point contact at the

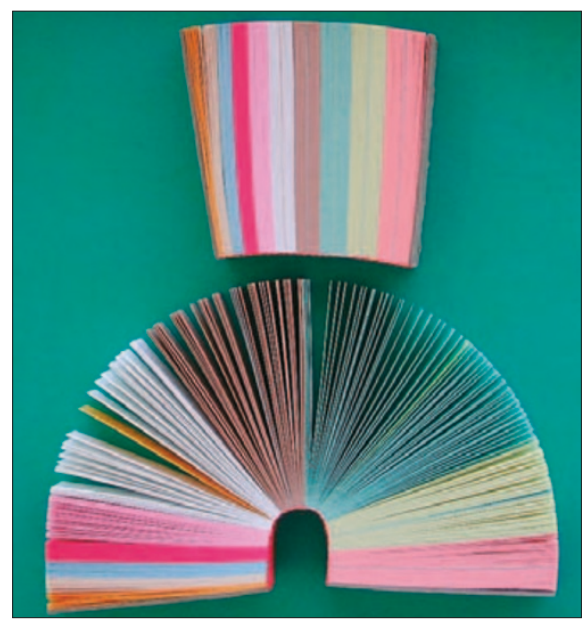

Fig. 11a A book of multi-coloured leaves which can be selectively used as a leaf gauge (Panadent Corp., CA, USA)

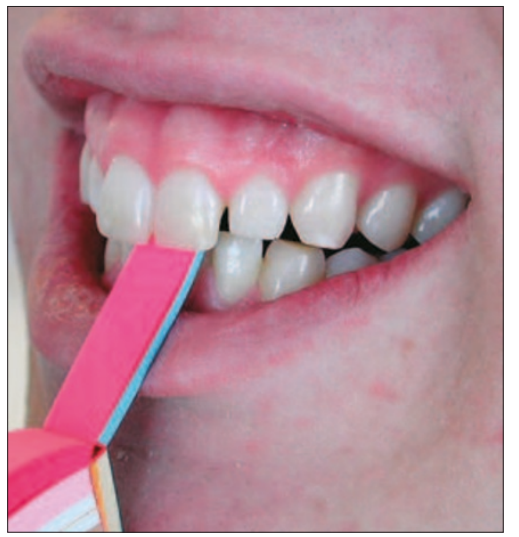

Fig. 11b A specific thickness of leaves has been selected in order to achieve an anterior reference point for registration of RCP

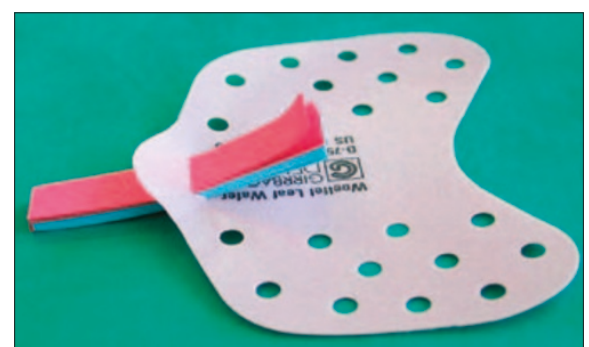

Fig. 11c $A$ support wafer can then be added to support the registration medium 
retruded position. The specially designed device (Girrbach Dental GmbH, Germany) has a graduated acetate bite platform, the position of which is adjusted antero-posteriorly until the teeth are minimally out of contact. A registration support wafer can then be added and the inter-dental record made (Fig. 12b).

\section{Power-centric registration method ${ }^{57}$}

This technique departs from the guided methods described so far in that the operator employs a directed force to achieve a retruded mandibular position. With the dentist standing in front and to the right of the supine patient, the left thumb and forefinger are placed over the upper teeth. The right thumb is placed on the superior aspect of the chin, while the second and third fingers take up position along the inferior border of the mandible. The operator's right arm is stiffened and pressure is applied from the shoulder by leaning. It has been suggested that reflex muscle shortening acts to retrude the mandible but it is likely that the mandible is pushed too far posteriorly, thus producing an error in RCP registration.

\section{COMPARISON OF RCP GUIDANCE \\ METHODS}

\section{Clinical variation in $\mathrm{RCP}$ recording}

It is important that the interocclusal record is made at the correct OVD using an accurate, dimensionally stable medium and an appropriate, well practised mandibular guidance technique. ${ }^{33}$ These principles should help the clinician make a retruded mandibular record which is accurate and reproducible. Helkimo ${ }^{22}$ suggested the range of clinical variation to be $0.07-0.11 \mathrm{~mm}$ antero-posteriorly, while Watson et al. ${ }^{58}$ found inter-clinician variation was much greater than intra-clinician variation when recording $\mathrm{CR}$ in edentulous patients. $\mathrm{McKee}^{59}$ echoed these findings and called for more undergraduate and postgraduate training in this important skill. Eriksson et $a l .{ }^{30}$ has investigated the clinical factors influencing the reproducibility of interocclusal recording methods in fixed, partial and complete prosthodontic cases. It was found that the dominant influence upon reproducibility was clinical technique rather than recording medium or the particular mandibular position recorded. Conversely, Utz et $a l .{ }^{60}$ has suggested that reproducibility is unachievable, regardless of technique or technical excellence. Utz et al. ${ }^{60}$ may be right in that absolute reproducibility of $\mathrm{RCP}$ is impossible, however differences of $0.08 \mathrm{~mm}$ have lead Posselt ${ }^{15}$ to conclude that these variations are 'probably clinically insignificant'.

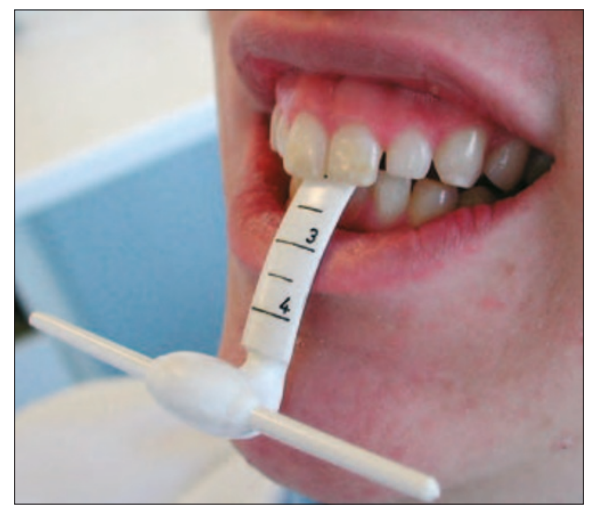

Fig. 12a An anterior clinical view showing the graduated acetate OSU Woelfel gauge (Girrbach Dental GmbH, Germany)

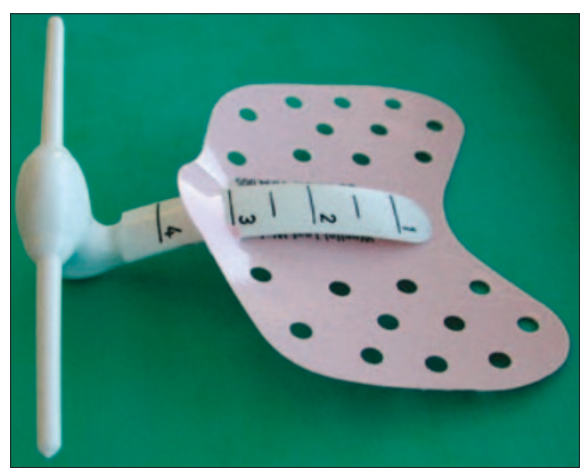

Fig. $12 \mathrm{~b}$ A registration support wafer can be used with the OSU Woelfel gauge

\section{Mandibular guidance studies}

The duplicability of gothic arch tracings has been studied in dentate subjects and found to be subject to diurnal variation. ${ }^{13,61,62}$ The same apex position could not be reproduced in any of the subjects and the greatest changes in position were in a mediolateral direction. This said, Helkimo $^{22}$ reported that guided mandibular closure gave more reproducible RCP recordings than no guidance, in both anteroposterior and mediolateral directions. Similar results were found by Kabcenell, ${ }^{16}$ who used bilateral manipulation, moderate and heavy pressure chin point guidance and the Schuyler technique. ${ }^{40}$ Moderate chin point guidance yielded the most consistent results. Kantor et al. ${ }^{64}$ investigated the influences of swallowing, chin-point guidance, Lucia jig, bilateral manipulation and the myo-monitor on the reproducibility of RCP recording. The most consistent guidance methods were the Lucia jig and bilateral manipulation, which have been demonstrated by electromyographic studies to produce the least temporalis and masseter activity. ${ }^{65}$ The myo-monitor and swallowing techniques were extremely variable. Recordings made using the myo-monitor have been shown to be anterior to RCP, variable between patients and variable on opposite sides of the same patient. ${ }^{66}$ The consistency of the Lucia jig combined with chin-point guidance or bilateral manipulation has been confirmed in other studies. ${ }^{34,67}$ In McKee's study ${ }^{59}$ the effect of training on mandibular guidance was evaluated. Prior to instruction, bimanual manipulation, chin point guidance and the Lucia jig produced similar results, with a spread of recorded positions made. After training in bilateral manipulation only, more consistent results (tolerance of $0.11 \mathrm{~mm}$ ) were achieved with it than with chin point guidance and a Lucia jig. The influence of patient-applied muscle force during the RCP recording must also be considered. Omar and Wise ${ }^{38}$ found mean flexure of the mandible, in the horizontal plane, to be $0.073 \mathrm{~mm}$ when patients apply maximal occlusal force.

\section{Anecdotal evidence}

Wise $^{25}$ described RCP location techniques for the dentate patient based upon the relative ease of mandibular manipulation. He classified patients as 'easy', 'manipulation with slight difficulty' and 'manipulation with more difficulty'. This classification is anecdotal and would certainly vary between clinicians, but might serve as a guide for RCP guidance technique selection, encouraging the dentist to examine mandibular movement more carefully. Wise suggested bimanual manipulation for easy patients. Anterior guidance from a tongue blade followed by bimanual manipulation was recommended for slightly more difficult patients. Very difficult patients required construction of a Lucia jig which may need to be left in situ for up to $30 \mathrm{~min}$ utes in order to disrupt proprioception. For some very difficult patients, proprioceptive deprogramming may need to be accomplished using an occlusal splint for an extended period. ${ }^{23,24,61}$

\section{SUMMARY}

RCP, that three-dimensional maxilloman-dibular relationship which results from initial teeth/tooth contact as the mandible hinges about the terminal hinge axis, is a relatively reproducible position. As a reproducible position, it is useful in the restorative management of dentate and edentulous individuals and as a reference point for the registration of transfer records, so that casts can be mounted on articulators. Although patients are reorganised into this position, it has been shown that a slide between RCP and ICP will be reintroduced after 2-12 years. ${ }^{28}$ This confirms that the occlusion is not constant but exhibits biological adaptability. Adaptability also means that 
accuracy to within the $0.08 \mathrm{~mm}$ cited by Posselt ${ }^{15}$ is probably clinically acceptable.

Establishing the RCP in the dentate patient and the retruded mandibular position in the edentulous patient can be difficult. Many factors can influence the accuracy and reproducibility of the recording including the patient, operator skill, the registration method employed, the material used for the recording and the time of the recording. It is unknown whether one registration method is better than another but it is the accuracy and reproducibility of achieving the retruded position in a given operator's hands which is probably of greatest importance. Training in mandibular guidance has been shown to produce constistency and it is recommended that all dental undergraduate students are schooled in this important skill.

Guiding the patient into the retruded position has been shown to give more consistent results than patient-guided techniques. From the published studies, well practised and executed chin point guidance, bimanual manipulation or use of an anterior jig is recommended. Deprogramming the masticatory system is required in some patients who have difficulty in achieving $\mathrm{RCP}$, and this is best done by an anterior jig or splint therapy.

Consistently identifying, recording and using the retruded mandibular position pays dividends for both the patient and operator. Restorative dental treatment can be provided successfully, in a controlled and accurate manner, with careful occlusal planning. Furthermore, treatments which have introduced occlusal disharmony can be rectified. Careful planning saves time during the treatment phase and increases patient and operator satisfaction. Ultimately the dentate or edentulous patient must be provided with an occlusion which is functional, aesthetic, stable, maintainable and does not cause discomfort.

The authors thank Dr J. D. Walter and Dr M. Fenlon, Guy's, King's \& St Thomas' Dental Institute, for their advice.

1. Davies S, Gray R M J. What is occlusion? Br Dent J 2001: 191: 235-245.

2. Davies S, Gray R M J. The examination and recording of the occlusion: why and how. Br Dent J 2001; 191: 291-302.

3. Keshvad A, Winstanley R B. An appraisal of the literature on centric relation. Part I. J Oral Rehab 2000; 27: 823-833.

4. Keshvad A, Winstanley R B. An appraisal of the literature on centric relation. Part II. J Oral Rehab 2000; 27: 1013-1023.

5. Keshvad A, Winstanley R B. An appraisal of the literature on centric relation. Part III. J Oral Rehab 2001; 28: 55-63.

6. Dixon D L. Overview of articulation materials and methods for the prosthodontic patient. J Prosthet Dent 2000; 83: 235-247.

7. Becker C M, Kaiser D A. Evolution of occlusion and occlusal instruments. J Prosthodont 1993; 2: 33-43.

8. Hall R E. Movements of the mandible. JAm Dent Assoc 1920; 7: 677-683.

9. Monson G S. Occlusion as applied to crown and bridgework. J Am Dent Assoc 1920; 7: 399-410.

10. Guichet N F. Applied gnathology: why and how. Dent Clin North Am 1969; 13: 687-700.

11. The Academy of Prosthodontics. Glossary of prosthodontic terms. J Prosthet Dent 1999; 81: 48-106.

12. The Academy of Prosthodontics. Glossary of prosthodontic terms. J Prosthet Dent 1994; 71: 40-116.

13. Grasso J E, Sharry J. The duplicability of arrowpoint tracings in dentulous subjects. J Prosthet Dent 1968; 20: 106-115.

14. Jasinevicius $T R$, Yellowitz J A, Vaughan G G, Brooks E $S$, Baughan L W, Cline N, Theiss L B. Centric relation definitions taught in 7 dental schools: results of faculty and student surveys. J Prosthodont 2000; 9: 87-94.

15. Posselt U. Studies in the mobility of the human mandible. Acta Odontol Scand 1952; 10: Suppl 10.

16. Kabcenell J L. Effect of clinical procedures on mandibular position. J Prosthet Dent 1964; 14: 266-278.

17. Ingervall $B$. Retruded contact position of the mandible. A comparison between children and adults. Odont Revy 1964; 15: 130-134.

18. Ingervall B. Studies of mandibular positions in children. Odont Revy 1968; 19: Suppl 15

19. Smith B G N. Occlusion: 1. General considerations. Dent Update 1991: 18: 141-145.

20. McCord J F, Grant A A. Registration: stage II intermaxillary relations. Br Dent J 2000; 188: 601-606.

21. Fenlon M R, Sherriff M, Walter J D. Association between the accuracy of intermaxillary relations and complete denture usage. J Prosthet Dent 1999; 81: 520-525.

22. Helkimo M. Prosthodontic treatment of partially edentulous patients. Various centric positions and methods of recording them. Zarb G A, Bergman $B$, Clayton J A, MacKay H F (eds) pp171-187. St Louis: CV Mosby, 1978.

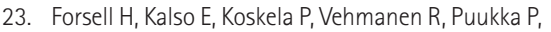
Alanen P. Occlusal treatments in temporomandibular disorders: a qualitative systematic review of randomised controlled trials. Pain 1999; 83: 549-561.

24. Dylina T J. A common-sense approach to splint therapy. J Prosthet Dent 2001; 86: 539-545.

25. Wise M D. Occlusion and restorative dentistry for the general practitioner. Part 2 - Examination of the occlusion and fabrication of study casts. Br Dent J 1982; 152: 160-165.

26. Ramfjord SP, Ash M M. Occlusion. 4rd edition, p 305 Philadelphia: WB Saunders Co, 1995.

27. Yamashita S, Hatch J P, Rugh J D. Does chewing performance depend upon a specific masticatory pattern? J Oral Rehab 1999: 26: 547-553.

28. Celenza FV. The centric position: replacement and character. J Prosthet Dent 1973; 30: 591-598.

29. Capp N J, Clayton J A. A technique for evaluation of centric relation tooth contacts. Part I - During normal temporomandibular joint function. J Prosthet Dent 1985; 54: 569-575.

30. Eriksson A, Öckert-Eriksson G, Lockowandt P, Eriksson 0 . Clincial factors and clinical variation influencing the reproducibility of interocclusal recording methods. Br Dent J 2002; 192: 395-400.

31. Shafagh $L$, Yoder $J$ L, Thayer K E. Diurnal variance of centric jaw position. J Prosthet Dent 1975; 34: 574-576.

32. Murray M C, Smith PW, Watts D C, Wilson N FH. Occlusal registration : science or art? Int Dent J 1999; 49: 41-46.

33. Warren K, Capp N. A review of principles and techniques for making interocclusal records for mounting working casts. Int J Prosthodont 1990; 3: 341-348

34. Öckert-Eriksson G, Eriksson A, Lockowandt P, Eriksson 0 . Materials for interocclusal records and their ability to reproduce a 3-dimensional jaw relationship. Int J Prosthodont 2000; 13: 152-158.

35. Kaplan R L. Concepts of occlusion and gnathology. Dent Clin NAm 1963: 1: 557-590.

36. Ingervall $B$, Helkimo M, Carlsson GE. Recording of the retruded position of the mandible with application of varying external pressure to the lower jaw in man.
Arch Oral Biol 1971; 16: 1165-1172

37. Lundeen $\mathrm{HC}$. Centric relation records: the effect of muscle action. J Prosthet Dent 1974; 31: 244-253.

38. Omar R, Wise M D. Mandibular flexure associated with applied muscle force in the retruded axis position. J Oral Rehab 1981; 8: 209-220.

39. Teo C S, Wise M D. Comparison of retruded axis articulator mountings with and without applied muscle force. J Oral Rehab 1981; 8: 363-376.

40. Schuyler C H. Intraoral method of establishing maxillomandibular relation. J Am Dent Assoc 1932; 19: 1012-1021.

41. Balthazar-Hart Y. Accuracy and dimensional stability of four interocclusal recording materials. J Prosthet Dent 1981; 45: 586-591.

42. Mullick S C, Stackhouse J A, Vincent G R V. A study of interocclusal record materials. J Prosthet Dent 1981; 46: 304-307.

43. Shanahan TE. Physiologic jaw relations and occlusion of complete dentures. J Prosthet Dent 1955; 5: 319-322.

44. Gysi A. The problem of articulation. Dent Cosmos 1910; 52: 1-19.

45. Fereday R C. Francis Balkwill and the physiology of mastication. Br Dent J 1994; 176: 386-393.

46. Jankelson B, Swain CW. Physiological aspects of masticatory muscle stimulation: the myomonitor. Quintessence Int 1972; 12: 57-62.

47. Jankelson B, Sparks S, Crane P F, Radke J C. Neural conduction of the myomonitor stimulus: a quantitative analysis. J Prosthet Dent $1975 ; \mathbf{3 4}$ : 245-253.

48. Baba K, Tsukiyama Y, Clark G T. Reliability, validity, and utility of various occlusal measurement methods and techniques. J Prosthet Dent 2000; 83: 83-89.

49. Lucia V 0 . Centric relation, theory and practice. J Prosthet Dent 1960; 10: 849-855.

50. Celenza FV. The theory and clinical management of centric positions II. Centric relation and centric relation occlusion. Int J Perio Rest Dent 1984; 4 62-66.

51. Dawson P E. Temporomandibular joint pain dysfunction problems can be solved. J Prosthet Dent 1973; 29: 100-104.

52. Smith B J. Implants in Clinical Dentistry. pp 224 London: Martin Dunitz, 2002.

53. Lucia $\vee 0$. Technique for recording centric relation J Pros Dent 1964; 14: 492-505.

54. Long $J \mathrm{H}$. Location of the terminal hinge axis by intraoral means. J Prosthet Dent 1970; 23: 11-24.

55. Long $\mathrm{J} \mathrm{H}$. Locating centric relation with a leaf gauge. J Prosthet Dent 1973; 29: 608-610.

56. Woelfel J B. New device for accurately recording centric relation. J Prosthet Dent 1986; 56: 716-727.

57. Roth R H. Functional occlusion for the orthodontist. Part I. J Clin Orth 1981: 15: 32-46.

58. Watson C J, Rowlands P W, Thomas S, Huggett $R_{t}$ Bates J F. The reproducibility of recording centric jaw relation in the edentulous patient. Quintessence Int 1987; 18: 35-40.

59. McKee J R. Comparing condylar position repeatability for standardized versus nonstandardized methods of achieving centric relation. J Prosthet Dent 1997; 77: 280-284.

60. Utz K H, Müller F, Lückerath W, Fuß E, Koeck B. Accuracy of check-bite registration and centric condylar position. J Oral Rehab 2002; 29: 458-466.

61. Boos R H. Physiologic denture technique. J Prosthet Dent 1956; 6: 726-740.

62. Boos R H. Intermaxillary relation established by biting power. J Am Dent Assoc 1940; 27: 1192-1199.

63. Walker $\mathrm{R} C$. A comparison of jaw relation recording methods. J Prosthet Dent 1962; 12: 685-694.

64. Kantor M E, Silvermann SI, Garfinkel L. Centric relation recording techniques: a comparative investigation. J Prosthet Dent 1972; 28: 593-600.

65. Hickman D M, Cramer R, Stauber W T. The effect of four jaw relations on electromyographic activity in human masticatory muscles. Arch Oral Biol 1993; 38: 261-264.

66. Noble W H. Antero-posterior position of myomonitor centric. J Prosthet Dent 1975; 33: 398-403.

67. Strohaver RA. A comparison of articulator mountings made with centric relation and myocentric position records. J Prosthet Dent 1972; 28: 378-390. 